\title{
Treatment Methods for Leakage Occurring at Staple Line After Laparoscopic Sleeve Gastrectomy
}

\author{
Varlik Erol $^{\mathrm{a}, \mathrm{c}}$, Cengiz Aydin ${ }^{\mathrm{a}}$, Levent Ugurlu ${ }^{\mathrm{a}}$, Omer Ozutemiz ${ }^{\mathrm{b}}$, \\ Mutlu Unver ${ }^{a}$, Safak Ozturk ${ }^{\mathrm{a}}$
}

\begin{abstract}
In this presentation, medical literature regarding this topic was reviewed along with the evaluation of treatment methods used in two specific cases during which leakages occurred along the staple line after laparoscopic sleeve gastrectomy (LSG). One of the two patients who developed a leakage after LSG was a 26-year-old female (case 1 ) and the other was a 20-year-old female (case 2). Case 1 was explored, when a leakage was diagnosed through clinical and screening procedures on day 3 after the surgery. After determining the leakage, the defect was primarily sutured and a feeding jejunostomy was placed. Case 1 developed leakage again on the fourth day after the second surgery, an esophagogastric stent was performed three times whose drainage was cut off from the leakage line, and the patient was discharged with full recovery. However, case 2 then developed leakage on the 14th day after surgery. Next an esophagogastric stent was placed in the patient, and a computer tomography (CT)-guided percutaneous drainage was placed to drain the collections inside the abdomen. Four weeks after this procedure, the stent was removed and after specifying that the leakage had closed, the patient was discharged with full recovery. The treatment methods of gastric leakages after LSG are also variable, and the treatment method is designated depending on the dimension of the leakage, the extent of the abdominal contamination, and the location of the leakage.
\end{abstract}

Keywords: Sleeve gastrectomy; Leakage; Treatment

\section{Introduction}

Obesity is a public health problem that affects both adults and

Manuscript accepted for publication June 23, 2015

aDepartment of General Surgery Clinic, Tepecik Education and Research Hospital, Izmir, Turkey

bepartment of Gastroenterology, School of Medicine, Ege University, Izmir, Turkey

${ }^{c}$ Corresponding Author: Varlik Erol, Department of General Surgery Clinic, Tepecik Education and Research Hospital, Yenisehir, Izmir, Turkey.

Email: varlikerol@gmail.com

doi: http://dx.doi.org/10.14740/jcs269w children. The frequency is gradually increasing globally and it contributes to the occurrence of many diseases. It is a major risk factor increasing morbidity and mortality. Today bariatric surgery is the most effective treatment method that maintains lasting weight loss especially for patients with comorbidity. Although laparoscopic sleeve gastrectomy (LSG) is a relatively new method used for the treatment of morbid obesity, it is a technique being used as a standard treatment for different grades of obese patients. Originally it was used as the first phase of a two-phase bariatric surgery for high-risk patients [1], but at present it is being applied as one of the basic bariatric. Clinical advantages result in a satisfactory amount of weight loss, do not cause any blockage (as a result of internal herniation) because no intestinal rotation is made, and eliminate risks of erosion and lapsing that are associated with the foreign bodies in treatment with gastric bands [2]. Along with the advantages, complications such as bleeding from the staple line, narrowness at the stomach residual and leakages at the staple line may occur. Leakages at the staple lines occur more frequently at the proximal staple line, at the distalis of the esophagogastric junction. In this presentation, the treatment methods in two cases of occurring leakages along the staple line after LSG were evaluated.

\section{Case Reports}

During the period from April 2013 to April 2014 of the 70 LSG cases carried out at the General Surgery Clinic of Tepecik Education and Research Hospital, two patients developed leakages at the staple line. The demographic parameters, applied treatment options, developing complications and treatment methods of these two patients were studied. LSG was applied to all patients diagnosed with morbid obesity. An upper gastrointestinal endoscopy was performed on all patients with the purpose of ruling out anatomic anomalies, mucosal pathologies, and helico-bacterial infection. The surgery was performed laparoscopically from five trocars. To standardize the width of the remnant stomach, a $34 \mathrm{~F}$ (French) thick dilatation tube was used at surgery. To resect the stomach, a laparoscopic stapler with the same brand and the same tissue closing thickness was used on all patients. Starting approximately $4-6 \mathrm{~cm}$ proximal to the stomach pylorus a dilatation tube guided resection towards the proximate. For hemostasis a laparoscopic clip and 


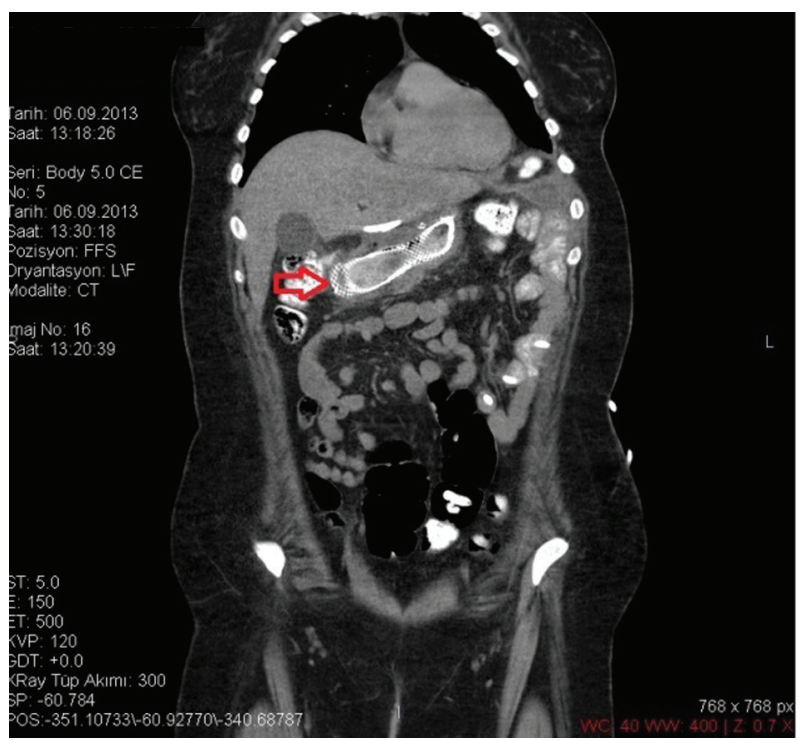

Figure 1. CT image of a stent placed after a leakage.

fibrin tissue glue were applied on the staple line at the end of the surgery. On the first day after surgery leakage control was completed by administering water-soluble opaque matter (by mouth) under fluoroscopy. Then the first day proceeded with water taken orally and the second day with liquid food.

\section{Case 1}

LSG was performed on a 26-year-old female patient based on diagnosis of morbid obesity. The body mass index (BMI) of the patient was determined as 40.3 before the surgery. The patient had no co-morbidity and no surgical history. Abdominal ultrasound and upper gastrointestinal system endoscopy were performed at the pre-operational stage and showed no pathology. The patient became tachycardic on the second day after the operation. The indication of peritonitis became clearer on the third day and when opaque matter extravasation was suspected after an abdominal computer tomography (CT) was taken. The

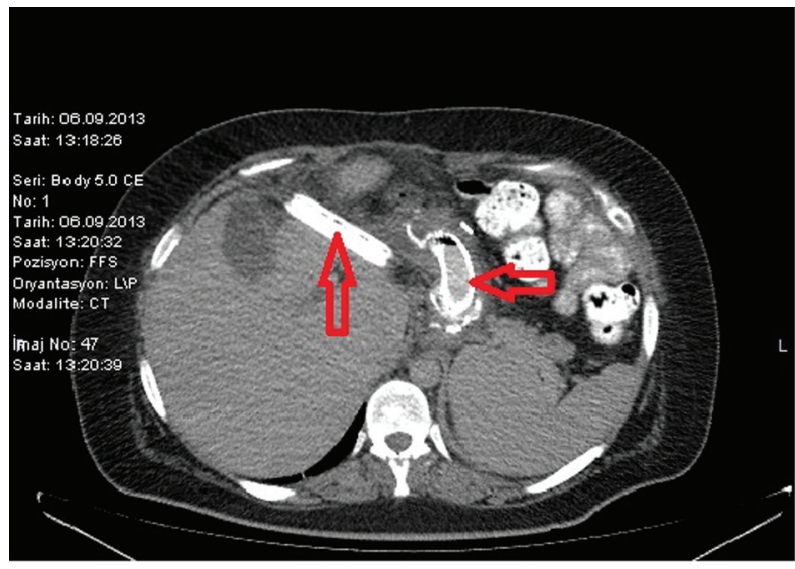

Figure 2. CT image of a stent placed after a leakage.

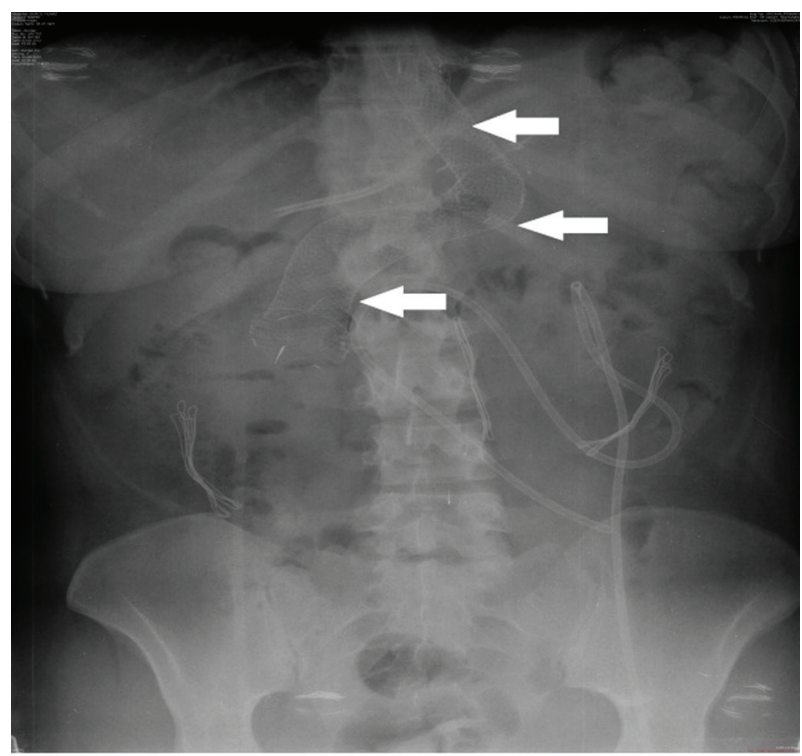

Figure 3. Direct radiography image of stent.

provisional diagnosis of a staple line leakage led to exploration of the patient on the third day post-procedure. After a leakage was determined near the esophagogastric junction, the defect was primarily sutured, a feeding jejunostomy catheter was placed and after inter-abdominal irrigation a drainage tube was placed. An appropriate antibiotics treatment was set out pursuant to the patient's inner abdominal fluid culture and blood culture results. On the fourth day after the second operation, a leakage redeveloped, so an esophagogastric stent was applied three times to the patient (Fig. 1-3). After the first stent was placed, chest radiography and thorax CT of the patient, who described respiratory distress, showed pleural effusion on the left side where upon a thorax drainage was placed and 6 days later when the symptoms receded the drainage was removed. While the stent was in place, oral intake continued. Because the patient vomited and complained of pain, both caused by the stent, a jejunostomy catheter provided feeding support and antiemetics were given along with analgesics for palliative purposes. After the stent was removed the drainage was

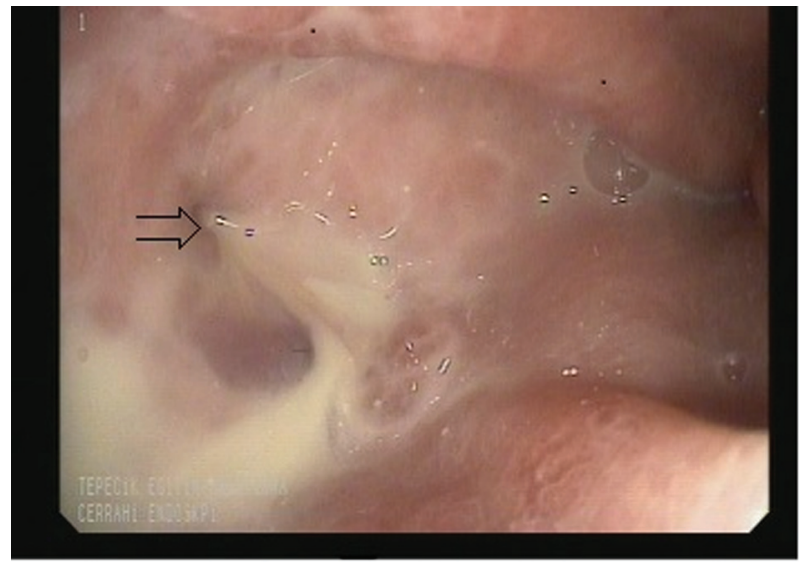

Figure 4. Image of a leakage determined by endoscopy. 
disconnected from the leakage line, oral intake of the patient was opened and the patient was discharged with full recovery.

\section{Case 2}

This is a 20-year-old female patient. The pre-operation BMI of the patient was determined as 45.2. On the 14th day after surgery, the CT of the abdomen, which was taken because the patient showed leukocytosis and fever, indicated minimal opaque extravasation along the stomach's proximal staple line and a collection indicative of an abscess of $6 \mathrm{~cm}$ at the same location. The endoscopy made after the pre-diagnosis of a leakage at the staple line, determined a staple line leakage about 0.5 $\mathrm{cm}$ at the distalis of the esophagogastric junction (Fig. 4) and in response an esophagogastric stent was applied to the patient. Additionally, a CT-guided percutaneous drainage was placed to drain the inner abdominal collection. As per the abscess and blood culture results we started an antibiotics treatment. While the stent was present the patient's oral intake was not closed. In the fourth week after the procedure, the stent was removed and an endoscopy immediately confirmed that the leakage had closed. It was also confirmed that the control CT of the patient did not show any signs of a leakage. Oral intake was started, the inner abdominal drainage was removed, and the patient was discharged with full recovery.

Both patients' post-operative examinations showed an absence of any additional complications. The 6 months examination showed that the first case incurred weight loss of $40 \mathrm{~kg}$ (BMI: 26.6) and the second case incurred weight loss of $35 \mathrm{~kg}$ (BMI: 30.3).

\section{Discussion}

Bariatric surgery is the most effective method, which procures satisfying weight loss and helps to maintain the ideal weight for patients with morbid obesity; also it is the most effective treatment in reducing co-morbidities related to obesity [3]. Compared to Roux-Y gastric bypass (RYGB) and duodenal switch (DS), LSG is a rather new surgery technique. Originally it was used in high-risk obese patients or patients who were planned to receive RYGB and DS, as a first step surgery method. But over time LSG has been proven to be an effective method for the patient to reach the ideal weight, and subsequently has become one of today's basic bariatric surgery methods. Compared to RYGB and DS, LSG is accepted as a lower risk procedure, although it can develop various complications, which increase morbidity and mortality. The most important complications related to stomach residue are hemorrhage, narrowness in the tubular stomach, and gastric leakage. Notwithstanding that the rates of occurring leakages are variable, it is reported as $0.7-20 \%$ [4]. The probability of a leakage amongst operations performed at our clinic is determined about $2.8 \%$. The treatment methods of gastric leakage are variable and are designated depending on the dimension of the leakage, the extent of the abdominal contamination, and the location of the leakage [5]. To determine a leakage at an early stage, patients at our clinic drink water-soluble opaque matter and are screened under fluoroscopy on the first day after surgery. However this method may be insufficient in determining subclinical leakages. Leakages developing secondarily to classical ischemia appear generally 5 - 6 days after the operation. Leakages developing because of technical reasons are determined usually on the first 2 days after the operation [6]. While clinical indications of patients developing leakages can be variable, the most common symptoms are: fever, epigastric pain, tachycardia and leukocytosis [7]. Our first case presented on the second day at first tachycardia. On the third day indications for peritonitis were determined. Methylene blue test, together with oral opaque matter under fluoroscopy and abdominal CT examinations may be helpful in determining leakages. Leakages determined at an early stage have an urgent surgical intervention indication, but most patients with late determination are managed with medical treatment [5]. For early stage leakages abdominal irrigation by laparoscopic exploration, a repair of the leakage if possible, and applying a jejunostomy for internal feeding is the most effective method. If the patient is not septic and if there is no extensive intra-abdominal fluid, percutaneous drainage of the internal fluid by placing an esophagogastric stent is usually sufficient in cases of late determined leakages. And if there is a presence of extensive intra-abdominal fluid and sepsis, performing an abdominal irrigation and feeding jejunostomy by surgical intervention and a repair of the leakage must be performed. As also mentioned in the literature, being one of the standard operations performed due to morbid obesity, LSG's most frequent indication is the choice of the patient [8]. Although patient satisfaction after LSG is very high, as it is also at our hospital, when a complication occurs (especially at the staple line) the recovery process is difficult. The most important factors of a leakage that may develop due to the operation technique are staple line ischemia and narrowing down the remnant stomach too much. Suturing the staple line in order to strengthen the line also reduces the risk of hemorrhage but the effect on the leakage is indeterminate [9]. Screening methods for the upper gastrointestinal system have a low sensitivity and specification for the determination of a leakage after surgery and therefore they are debateable [10]. According to our facts it was not possible to determine a leakage under fluoroscopy with water-soluble opaque matter alone.

In both cases, the suspicion of a leakage was confirmed by taking a CT together with orally administered opaque matter.

In cases where indications are determined at a post-operational early stage (especially indications like tachycardia without peritonitis, fever, leukocytosis), the leakage must be excluded without wasting time. If the indications continue although a leakage was not determined by $\mathrm{CT}$, a laparoscopic exploration should be performed without any hesitation.

Leakages, which cannot be repaired with surgery or the treatment of retarded leakages after LSG, esophagogastric stent and percutaneous drainage method must be performed. After placing the stent the patient can continue with oral intake. After the stent has remained at least 4 weeks it must be removed from the leakage line and controlled by endoscopy. Within this period the antibiotic therapy must be arranged according to the results of the cultures. It is also important to pay attention to the daily calorie ration and to ensure that the liquid 
electrolyte support is sufficient. In order to provide all of these conditions, in our opinion it is necessary that the treatment is multi-disciplinary and to be performed with a team that has experience with morbid obesity.

\section{Conflict of Interests}

The authors declare that there is no conflict of interests regarding the publication of this article.

\section{References}

1. Regan JP, Inabnet WB, Gagner M, Pomp A. Early experience with two-stage laparoscopic Roux-en-Y gastric bypass as an alternative in the super-super obese patient. Obes Surg. 2003;13(6):861-864.

2. Nguyen NT, Nguyen XM, Dholakia C. The use of endoscopic stent in management of leaks after sleeve gastrectomy. Obes Surg. 2010;20(9):1289-1292.

3. Gastrointestinal surgery for severe obesity: Proceedings of a National Institutes of Health Consesus Development Conference. Am J Clin Nutr. 2002;55(2):487-619.

4. Tan JT, Kariyawasam S, Wijeratne T, Chandraratna HS.
Diagnosis and management of gastric leaks after laparoscopic sleeve gastrectomy for morbid obesity. Obes Surg. 2010;20(4):403-409.

5. Abraham A, Rizvon K, Singh J, Siddiqui G, Prasad A, Rashid S, Vardaros M, et al. Successful management of a gastric sleeve leak with an endoscopic stent. Case Rep Gastrointest Med. 2012;2012:205979.

6. Marquez MF, Ayza MF, Lozano RB, Morales Mdel M, Diez JM, Poujoulet RB. Gastric leak after laparoscopic sleeve gastrectomy. Obes Surg. 2010;20(9):1306-1311.

7. Csendes A, Braghetto I, Leon P, Burgos AM. Management of leaks after laparoscopic sleeve gastrectomy in patients with obesity. J Gastrointest Surg. 2010;14(9):1343-1348.

8. Brethauer SA, Hammel JP, Schauer PR. Systematic review of sleeve gastrectomy as staging and primary bariatric procedure. Surg Obes Relat Dis. 2009;5(4):469-475.

9. Chen B, Kiriakopoulos A, Tsakayannis D, Wachtel MS, Linos D, Frezza EE. Reinforcement does not necessarily reduce the rate of staple line leaks after sleeve gastrectomy. A review of the literature and clinical experiences. Obes Surg. 2009;19(2):166-172.

10. Gagner M, Deitel M, Kalberer TL, Erickson AL, Crosby RD. The Second International Consensus Summit for Sleeve Gastrectomy, March 19-21, 2009. Surg Obes Relat Dis. 2009;5(4):476-485. 